\title{
Optimal Design of Tolerance Ring in Double-Decker Catcher Bearing System
}

\author{
Zhu Yili* and Zhang Yongchun
}

Department of Electrical Engineering, Changzhou Institute of Technology, Changzhou, Jiangsu, 213002, P.R. China

\begin{abstract}
In an active magnetic bearing (AMB) system, the catcher bearings (CBs) are essential to protect the system in cases of AMB failure. On the basis of the former researches of double-decker catcher bearings (DDCBs), a tolerance ring is proposed to further improve the performance of DDCB. Firstly, the support model of DDCB supported by tolerance ring is established. Then numerical simulations are carried out to determine the design parameters of tolerance ring. Finally, the tolerance ring is manufactured according to the obtained parameters and relative experiments are carried out to verify the theoretical analysis results. Both theoretical and experimental results validated that proper tolerance ring is beneficial to reduce the following impact forces and vibrations after rotor drop.
\end{abstract}

Keywords: Active magnetic bearing, catcher bearings, double-decker catcher bearing, rotor drop, tolerance ring.

\section{INTRODUCTION}

There are many advantages of AMB compared with traditional mechanical bearings, such as no friction, no lubrication as well as adjustable support stiffness and damping. However, $\mathrm{CBs}$ are indispensable to protect the AMB assembly, for they can temporarily support the rotor during maintenance and prevent destructions of the system after a possible AMB failure.

Most of the former researches focused on the dynamic responses after rotor drop. Xie and Flowers [1] numerically investigated the steady-stator behaviors of a rotor drops onto $\mathrm{CB}$ and studied the influences of rotor imbalance, support stiffness and damping on rotor dynamic responses. Cole et al. [2] use a series of flexible beams to analyze the influences of different parameters on the elastic deformation of the inner race and ball load distributions. However, rotor drop simulations were not conducted. Wang and Noah [3] analyzed the steady-state responses of a rigid rotor drops onto a positive clearance bush using the fixed-point algorithm and predicted a chaotic whirling of the rotor. Also some other researchers focused on new type of $\mathrm{CBs}$, such as zero clearance catcher bearing [4] and hybrid air foil catcher bearing [5]. And Reference [6] studied the use of a DDCB composed of two deep groove inner ball bearings and one deep groove outer ball bearing. The results showed that the use of DDCB can to some extent reduce the following vibrations, heating and impact forces after rotor drop.

In order to further improve the working performances of DDCB, tolerance ring is proposed to be installed in the DDCB system. Detailed collision model and support model of DDCB with tolerance ring are established. Combining the rigid model and AMB support model established in reference
[6], theoretical simulations are carried out to obtain optimum tolerance ring design parameters. Then the tolerance ring is manufactured using those obtained parameters. The advantages of using tolerance ring are verified by both simulations and experiments.

\section{SIMULATION}

\subsection{Structure of AMB System}

Fig. (1) shows the studied structure of a motor drive system equipped with magnetic bearings, where 1: rotor; 2 : radial displacement sensor; 3: axial displacement sensor; 4: catcher bearing; 5: axial magnetic bearing; 6: radial magnetic bearing; 7: motor. The motor is located between the two radial magnetic bearings. Each radial magnetic bearing generates radial forces. Axial magnetic bearings regulate the axial forces in the shaft direction. Besides those magnetic bearings, two catcher bearings are located in the two ends of the structure respectively to prevent damages after rotor drop. The air gap between the catcher bearing inner race and the rotor is half of the air gap of AMB.

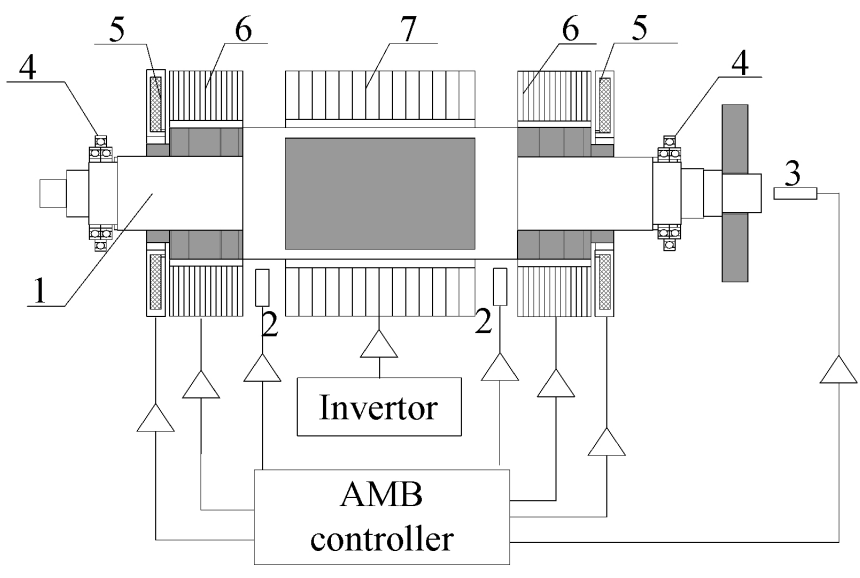

Fig. (1). Structure of AMB system. 
The structure of DDCB installed tolerance ring is shown in Fig. (2). With the purpose of improving the rotational speed of outer bearing can share, the inner layer of DDCB has two deep groove ball bearings, and the outer layer has only one ball bearing. The adapter ring rigidly connects the outer ring of inner bearings and inner ring of outer bearing forming the intermediate race. The tolerance ring is installed between the bearing house and foundation support. And there is a restrict gap $(0.05 \mathrm{~mm})$ to restrict the displacement of bearing house once the tolerance ring deforms too large.

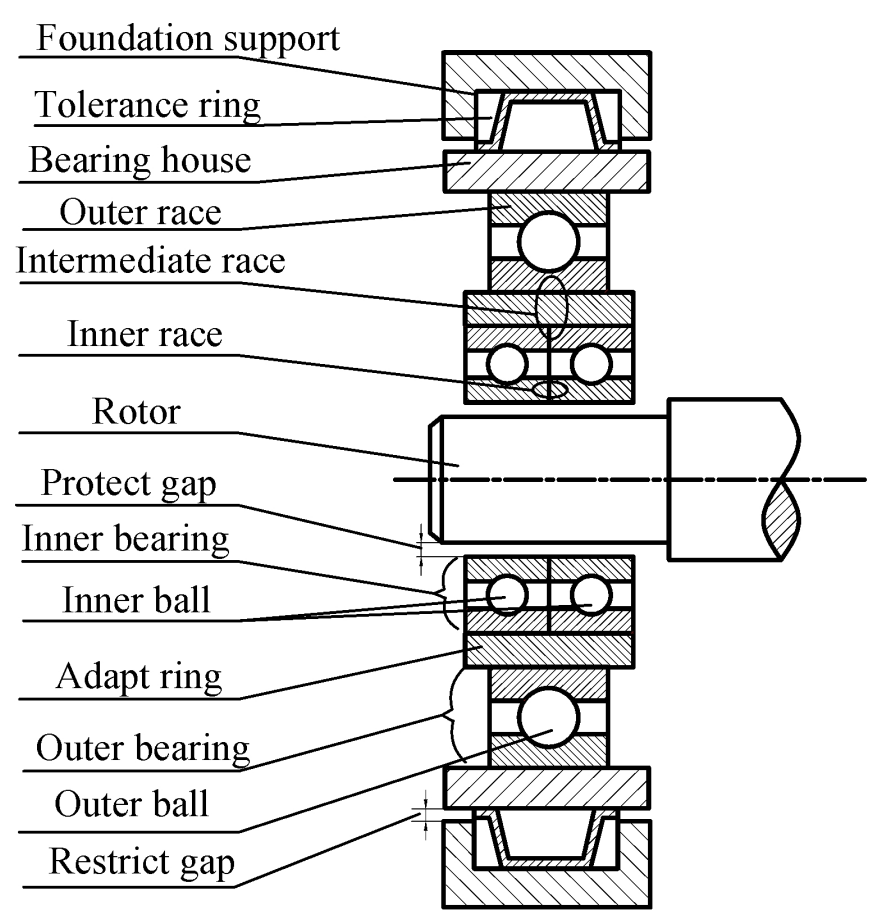

Fig. (2). Structure of analyzed DDCB.

\subsection{Simulation Model}

According to the structure of AMB system, the rotor force model can be obtained, as shown in Fig. (3). When the AMB system is in normal operation, the rotor bears left-hand and right-hand magnetic bearing forces $\left(F_{\text {a1x }}, F_{\text {aly }}\right.$ and $F_{\text {a2x }}$, $\left.F_{\text {a2y }}\right)$, centrifugal forces $\left(F_{\mathrm{cx}}, F_{\mathrm{cy}}\right)$ and gravity $\left(G_{\mathrm{r}}\right)$ respectively. After AMB system failure, the rotor bears lefthand and right-hand $\mathrm{CB}$ forces $\left(F_{\mathrm{b} 1 \mathrm{x}}, F_{\mathrm{b} 1 \mathrm{y}}\right.$ and $\left.F_{\mathrm{b} 2 \mathrm{x}}, F_{\mathrm{b} 2 \mathrm{y}}\right)$, centrifugal forces $\left(F_{\mathrm{cx}}, F_{\text {cy }}\right)$ and gravity $\left(G_{\mathrm{r}}\right)$ respectively.

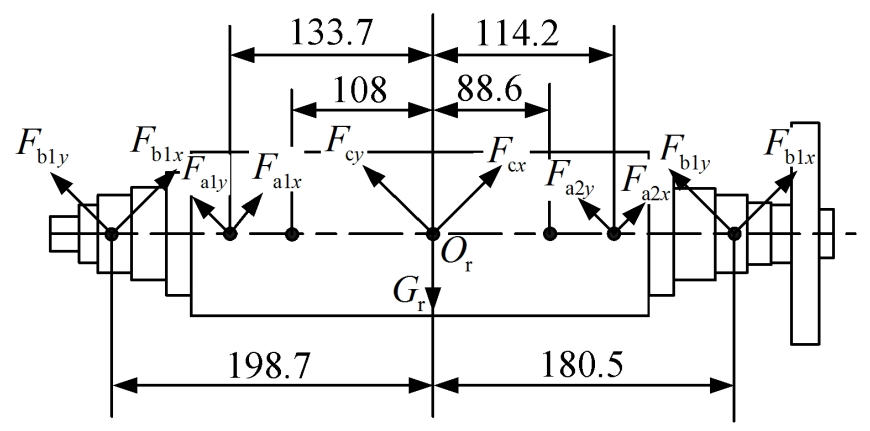

Fig. (3). Rotor force model.

Then the rotor motion equation can be written as:

$m \ddot{x}+G \dot{x}=A F_{\mathrm{a}}+B F_{\mathrm{b}}+F_{\mathrm{c}}+F_{\mathrm{g}}$
Where mass matrix $\boldsymbol{m}=\operatorname{diag}\left(m_{\mathrm{r}}, m_{\mathrm{r}}, J, J\right), m_{\mathrm{r}}$ is rotor mass, $J$ is rotor transverse moment of inertia (MOI), barycenter displacement $\boldsymbol{x}=\left(x_{\mathrm{r}}, y_{\mathrm{r}}, \theta_{x}, \theta_{y}\right)^{\mathrm{T}}, x_{\mathrm{r}}$ and $y_{\mathrm{r}}$ are the displacements of barycenter in the direction of $x$ and $y$ axis respectively, $\theta_{\mathrm{x}}$ and $\theta_{\mathrm{y}}$ are the rotational displacement of barycenter around the direction of $x$ and $y$ axis respectively. $G$ is the gyroscopic torque matrix, $\boldsymbol{G}=\left(\begin{array}{cccc}0 & 0 & 0 & 0 \\ 0 & 0 & 0 & 0 \\ 0 & 0 & 0 & \omega J_{z} \\ 0 & 0 & -\omega J_{z} & 0\end{array}\right), J_{\mathrm{z}}$ is rotor polar MOI, $\omega$ is the rotor angular velocity. $\boldsymbol{A}$ and $\boldsymbol{B}$ are the introduced coefficient matrixes,

$$
\begin{aligned}
& \boldsymbol{A}=\left(\begin{array}{cccc}
1 & 0 & 1 & 0 \\
0 & 1 & 0 & 1 \\
0 & l_{\mathrm{a} 1} & 0 & -l_{\mathrm{a} 2} \\
-l_{\mathrm{a} 1} & 0 & l_{\mathrm{a} 2} & 0
\end{array}\right), \\
& \boldsymbol{B}=\left(\begin{array}{cccc}
1 & 0 & 1 & 0 \\
0 & 1 & 0 & 1 \\
0 & l_{\mathrm{b} 1} & 0 & -l_{\mathrm{b} 2} \\
-l_{\mathrm{b} 1} & 0 & l_{\mathrm{b} 2} & 0
\end{array}\right) ;
\end{aligned}
$$

magnetic force vector $\boldsymbol{F}_{\mathrm{a}}=\left(F_{\mathrm{a} 1 x}, F_{\mathrm{a} 1 y}, F_{\mathrm{a} 2 x}, F_{\mathrm{a} 2 y}\right)^{\mathrm{T}}$; rotor centrifugal force vector $\boldsymbol{F}_{\mathrm{a}}=\left(F_{c x}, F_{c y}, 0,0\right)^{\mathrm{T}}$; rotor gravity vector $\boldsymbol{F}_{g}=\left(F_{g x}, F_{g y}, 0,0\right)^{\mathrm{T}}$.

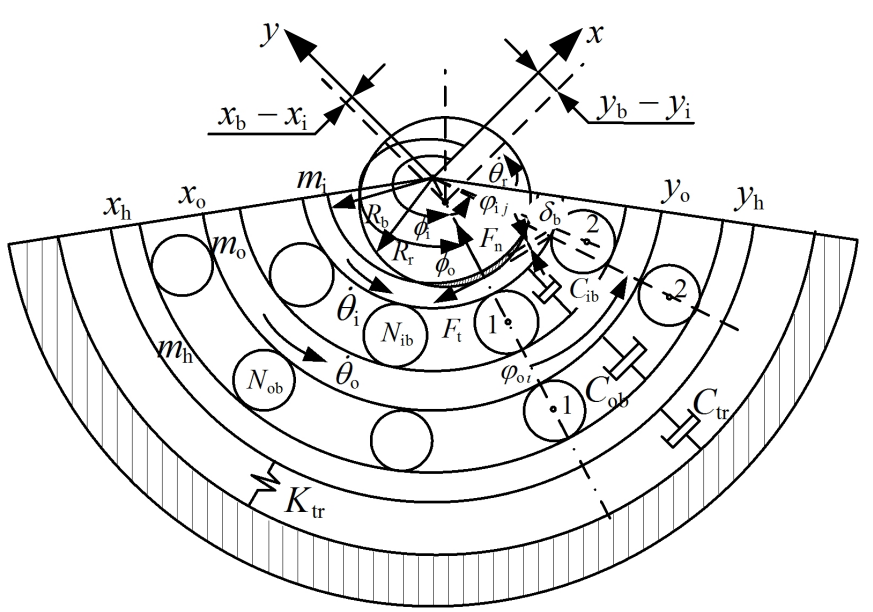

Fig. (4). Contact model after rotor drop.

The contact model after rotor drops onto the DDCB supported by tolerance ring is shown in Fig. (4). Here the model of tolerance ring is seems as stiffness $K_{\text {tr }}$ and damping $C_{\mathrm{tr}}$, which will be detailed analyzed in the following part. $F_{\mathrm{n}}$ and $F_{\mathrm{t}}$ are the normal impact force and tangential frictional force between the rotor and inner race, respectively; $R_{\mathrm{r}}$ and $R_{\mathrm{b}}$ are the shaft radius and inner race bore radius at the CB position, respectively; $m_{\mathrm{i}}$ and $m_{\mathrm{o}}$ are the mass of the inner 
race and intermediate race respectively. And the DDCB outer race is rigidly installed in the bearing house, the whole mass is $m_{\mathrm{h}} ; C_{\mathrm{ib}}$, and $C_{\mathrm{ob}}$ are the support damping of the inner bearing and outer bearing respectively; $x_{\mathrm{b}}, x_{\mathrm{i}}, x_{\mathrm{o}}$ and $x_{\mathrm{h}}$ are the vibration displacements of the rotor, inner race, intermediate race and outer race in the $x$-axis, respectively; $y_{\mathrm{b}}, y_{\mathrm{i}}, y_{\mathrm{o}}$ and $y_{\mathrm{h}}$ are the vibration displacements of the rotor, inner race, intermediate race and outer race in the $y$-axis, respectively; $\phi_{\mathrm{i}}$ is the defined rotor-inner race contact angle. To simplify the analysis, it is assumed that there always exists a inner and outer ball labeled as " 1 " in the direction of relative displacement, so the $j$ th inner ball position angle is $\varphi_{i j}=2 \pi(j-1) / N_{i \mathrm{~b}}$, and the $\boldsymbol{l}$ th outer ball position angle is $\varphi_{o l}=2 \pi(l-1) / N_{\mathrm{ob}}$, where $N_{\mathrm{ib}}$ and $N_{\mathrm{ob}}$ are the ball number of the single inner bearing and outer bearing respectively. $\theta_{\mathrm{r}}$, $\theta_{i}$ and $\theta_{\mathrm{o}}$ are the angular displacements of the rotor, inner race and intermediate race respectively, and $\delta_{\mathrm{b}}$ is the penetration depth between the rotor and inner race.

The radial motion equation for the new type DDCB after rotor drop is given by

$$
\begin{aligned}
& \boldsymbol{m}_{\mathrm{b}} \ddot{\boldsymbol{x}}_{\mathrm{b}}+\boldsymbol{C}_{\mathrm{b}} \dot{\boldsymbol{x}}_{\mathrm{b}}=\boldsymbol{F}_{\mathrm{b}}+\boldsymbol{F}_{\mathrm{d}}+\boldsymbol{F}_{\mathrm{h}}-\boldsymbol{F}_{\mathrm{rb}} \\
& \text { where } \boldsymbol{m}_{\mathrm{b}}=\operatorname{diag}\left(m_{\mathrm{i}}, m_{\mathrm{i}}, m_{\mathrm{o}}, m_{\mathrm{o}}, m_{\mathrm{h}}, m_{\mathrm{h}}\right) \text {; } \\
& \boldsymbol{x}_{\mathrm{b}}=\left(x_{\mathrm{i}}, y_{\mathrm{i}}, x_{\mathrm{o}}, y_{\mathrm{o}}, x_{\mathrm{h}}, y_{\mathrm{h}}\right)^{\mathrm{T}} \text {; } \\
& \boldsymbol{F}_{\mathrm{b}}=\left(F_{\mathrm{ii} x}, F_{\mathrm{ii} y}, F_{\mathrm{ie} x}+F_{\text {oi } x}, F_{\text {ie } y}+F_{\text {oi } y}, F_{\text {oe } x}, F_{\text {oe } y}\right)^{\mathrm{T}} \text {; } \\
& \boldsymbol{F}_{\mathrm{rb}}=\left(F_{\mathrm{b} x}, F_{\mathrm{b} y}, 0,0,0,0\right)^{\mathrm{T}} ; \boldsymbol{F}_{\mathrm{d}}=\left(0,0,0,0, F_{\mathrm{tr} x}, F_{\mathrm{tr} y}\right)^{\mathrm{T}} ; \\
& \boldsymbol{F}_{\mathrm{h}}=\left(0,0,0,0, F_{\mathrm{h} x}, F_{\mathrm{h} y}\right)^{\mathrm{T}} \\
& \boldsymbol{C}_{\mathrm{b}}=\left[\begin{array}{cccccc}
2 C_{\mathrm{ib}} & 0 & -2 C_{\mathrm{ib}} & 0 & 0 & 0 \\
0 & 2 C_{\mathrm{ib}} & 0 & -2 C_{\mathrm{ib}} & 0 & 0 \\
-2 C_{\mathrm{ib}} & 0 & 2 C_{\mathrm{ib}}+C_{\mathrm{ob}} & 0 & 0 & 0 \\
0 & -2 C_{\mathrm{ib}} & 0 & 2 C_{\mathrm{ib}}+C_{\mathrm{ob}} & 0 & 0 \\
0 & 0 & -C_{\mathrm{ob}} & 0 & C_{\mathrm{ob}}+C_{\mathrm{tr}} & 0 \\
0 & 0 & 0 & -C_{\mathrm{ob}} & 0 & C_{\mathrm{ob}}+C_{\mathrm{tr}}
\end{array}\right] ;
\end{aligned}
$$

In the above equations, $F_{\text {ii } x}, F_{\text {ii } y}, F_{\text {ie } x}, F_{\text {oi } x}, F_{\text {oe } x}, F_{\text {oe } y}$ are the contact forces of balls acting on the relevant races; $F_{\mathrm{b} x}$ and $F_{\mathrm{b} y}$ are the contact forces of rotor acting on the inner race in the $x$ and $y$ directions respectively; $F_{\mathrm{h} x}$ and $F_{\mathrm{h} y}$ are the contact forces of foundation support acting on the bearing house in the $x$ and $y$ directions respectively.

Each protuberance of tolerance ring can be seemed as a spring with the stiffness of $K_{\text {tr }}$, as shown in Fig. (5). And $N_{\text {tr }}$ is the number of protuberances.

The stiffness can be calculated as [7]:

$$
K_{\mathrm{tr}} \approx 4.8 E_{\mathrm{tr}} w_{\mathrm{tr}}\left(\frac{B_{\mathrm{tr}}}{D_{\mathrm{ptr}}}\right)^{3}
$$

where $E_{\mathrm{tr}}$ is the elasticity modulus of tolerance ring; $w_{\mathrm{tr}}$ is the width of protuberance; $B_{\mathrm{tr}}$ is the material thickness; $D_{\mathrm{ptr}}$ is the pitch diameter of protuberance, and $D_{\mathrm{ptr}} \approx \pi d_{\mathrm{tr}} / N_{\mathrm{tr}}, d_{\mathrm{tr}}$ is the diameter of tolerance ring.

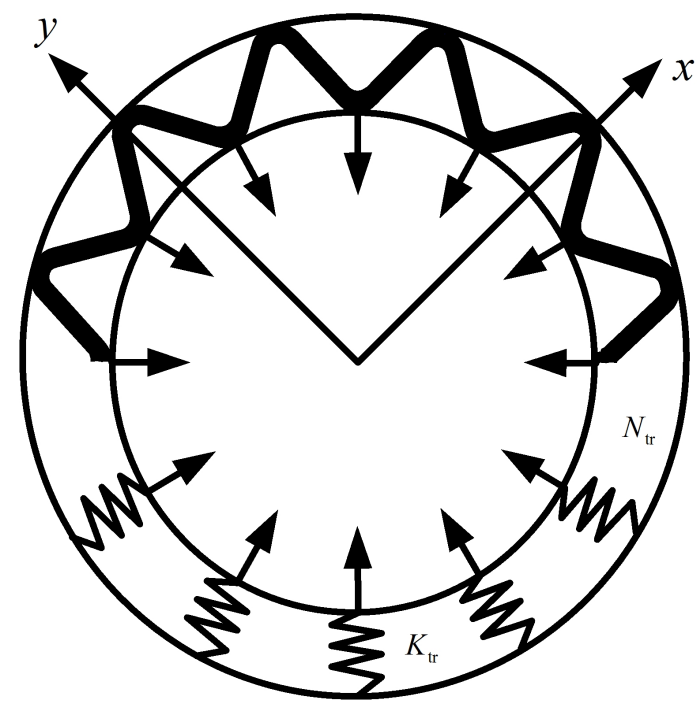

Fig. (5). Support model of tolerance ring.

Then the elastic force of tolerance ring acting on the bearing house in $x$ and $y$ directions can be calculated as:

$$
\left\{\begin{array}{l}
F_{\mathrm{tr} x}=\sum_{\xi=1}^{N_{\mathrm{tr}}}\left[K_{\mathrm{tr}}\left(\sigma_{\mathrm{tr} \xi}\right)_{+} \cos \left(\phi_{\mathrm{h}}+\varphi_{\mathrm{tr} \xi}\right)\right] \\
F_{\mathrm{tr} y}=\sum_{\xi=1}^{N_{\mathrm{tr}}}\left[K_{\mathrm{tr}}\left(\sigma_{\mathrm{tr} \xi}\right)_{+} \sin \left(\phi_{\mathrm{h}}+\varphi_{\mathrm{tr} \xi}\right)\right]
\end{array}\right.
$$

where the subscript " + " denotes that $\sigma_{\text {tr } \xi}=0$ when protuberance deformation $\sigma_{\mathrm{tr} \xi} \leq 0$.

\subsection{Simulation Results}

The whole simulation composes two parts, rotor motions before and after AMB failure. Firstly, the rotor motions during normal operation are simulated as the initial conditions for the dynamic simulations after rotor drop. During the simulation, based on relevant AMB theory, hertz contact theory [8] and well as ball bearing support theory, the AMB forces and DDCB forces are calculated using the real time rotor and DDCB motions. The chosen inner and outer ball bearing types are 61905 and 61810 respectively. The diameter and width of tolerance ring are firstly confirmed according to the specific mechanical structure. Then protuberant number and material thickness are modified to optimize the performance of tolerance ring. And the other relevant simulation parameters are listed in Table $\mathbf{1 .}$

The maximum impact forces between rotor and inner race after rotor drop for different tolerance ring parameters at the initial rotor rotational speed $12000 \mathrm{r} / \mathrm{min}, 18000 \mathrm{r} / \mathrm{min}$, $24000 \mathrm{r} / \mathrm{min}$ and $30000 \mathrm{r} / \mathrm{min}$ can be calculated after obtaining the rotor and DDCB motions, as shown in Fig. (6). Focusing on the working performances of tolerance ring in high-speed situations, it is advisable to choose protuberant number 45 and material thickness $0.4 \mathrm{~mm}$. 
Table 1. Relevant simulation parameters.

\begin{tabular}{|l|c|}
\hline \multicolumn{1}{|c|}{ Parameter } & Value \\
\hline \hline Rotor unbalance $e_{\mathrm{r}}(\mu \mathrm{m})$ & 2.5 \\
\hline Rotor transverse MOI $J\left(\mathrm{~kg} \cdot \mathrm{mm}^{2}\right)$ & $6.1 \mathrm{e} 5$ \\
\hline Rotor polar MOI $J_{\mathrm{z}}(\mathrm{kg} \cdot \mathrm{mm} 2)$ & $4.7 \mathrm{e} 3$ \\
\hline Mass of the inner race $m_{\mathrm{i}}(\mathrm{kg})$ & $16.4 \mathrm{e}-3$ \\
\hline Mass of the intermediate race $m_{\mathrm{o}}(\mathrm{kg})$ & 0.23 \\
\hline Mass of the bearing house $m_{\mathrm{h}}(\mathrm{kg})$ & 0.53 \\
\hline Current stiffness of AMB $k_{\mathrm{i}}(\mathrm{N} / \mathrm{A})$ & 166.5 \\
\hline Displacement stiffness of AMB $k_{\mathrm{x}}(\mathrm{N} / \mathrm{m})$ & $1.25 \mathrm{e} 6$ \\
\hline Protective gap of ABs $c_{\mathrm{r} 1}(\mathrm{~mm})$ & 0.125 \\
\hline Mass of rotor $m_{\mathrm{r}}(\mathrm{kg})$ & 9.1 \\
\hline Material elasticity modulus of tolerance ring $E_{\mathrm{tr}}(\mathrm{GPa})$ & 187.5 \\
\hline Tolerance ring damping $C_{\mathrm{tr}}\left(\mathrm{N} \cdot \mathrm{s} \cdot \mathrm{m}^{-1}\right)$ & 0 \\
\hline Tolerance ring diameter $d_{\mathrm{tr}}(\mathrm{mm})$ & 85 \\
\hline Width of protuberance $w_{\mathrm{tr}}(\mathrm{mm})$ & 25 \\
\hline
\end{tabular}

\section{EXPERIMENTS}

Using the parameters obtained above, two types of tolerance ring are manufactured by Saint-Gobain Company, and type "A": protuberance number 43, material thickness $0.4 \mathrm{~mm}$; type "B": protuberance number 43, material thickness $0.5 \mathrm{~mm}$. Fig. (7) shows the related parts used in the analyzed DDCB system. Fig. (8) presents the rotor drop test rig, where 1:PC; 2: AMB system controller; 3: high-speed magnetic levitation motor; 4: inverter; 5: Labview data acquisition (DAQ) boards; 6: software of data acquisition system. The displacement sensor signals are collected by Labview DAQ boards and saved in the PC. A subsequent analysis of the collected data is carried out using MATLAB software.

Using the collected displacement sensor signals, the rotor orbits $0.1 \mathrm{~s}$ before rotor drop and $0.2 \mathrm{~s}$ after rotor drop onto different types of DDCB at the initial rotor speed 12000 $\mathrm{r} / \mathrm{min}$ can be obtained, as shown in Fig. (9). It can be seen that the use of tolerance ring help to reduce the rotor vibration amplitudes after rotor drop, and the work performance of type "A" is much better.

Once the rotor vibrations after rotor drop are obtained by the experiments, using equation (1) the support forces of DDCBs can be calculated as:

$$
\boldsymbol{F}_{\mathrm{b}}=\operatorname{inv}\left(\boldsymbol{m} \ddot{\boldsymbol{x}}+\boldsymbol{G} \dot{\boldsymbol{x}}-\boldsymbol{F}_{\mathrm{c}}-\boldsymbol{F}_{\mathrm{g}}\right)
$$

The maximum impact forces calculated using the experimental results $0.5 \mathrm{~s}$ after rotor drops are shown in Fig. (10). The results indicate that tolerance ring can effectively reduce the impact forces after rotor drop, and the effects of type "A" are much better. (a) $12000 \mathrm{r} / \mathrm{min}$

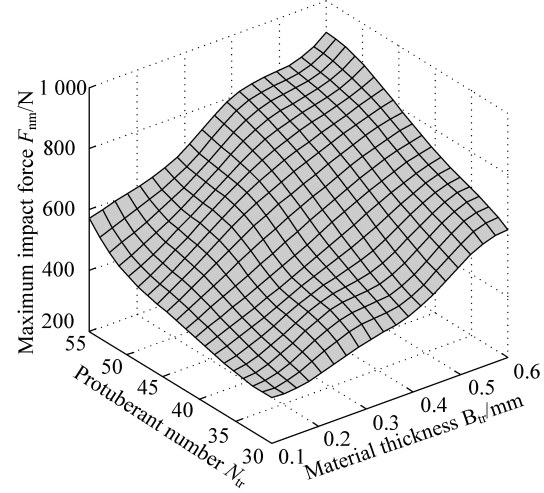

(b) $18000 \mathrm{r} / \mathrm{min}$

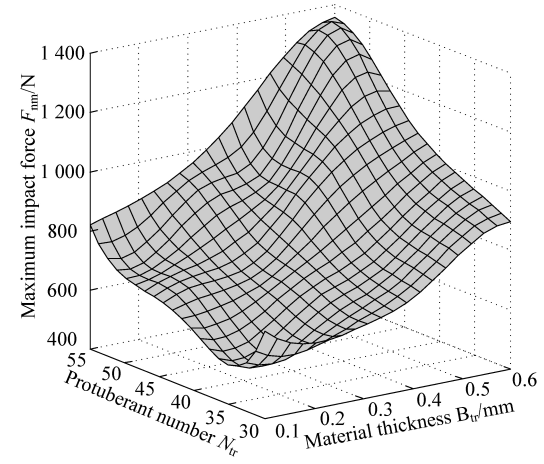

(c) $24000 \mathrm{r} / \mathrm{min}$

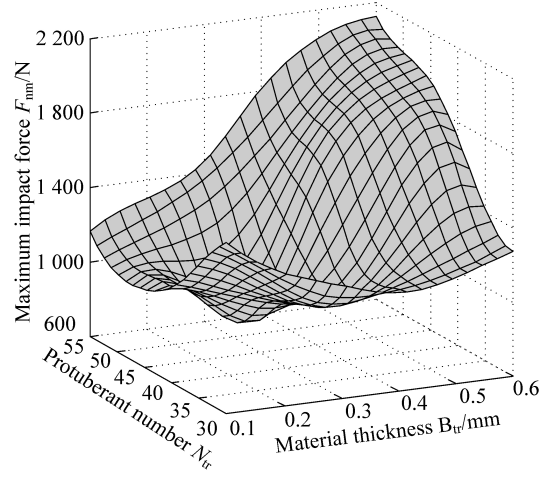

(d) $30000 \mathrm{r} / \mathrm{min}$

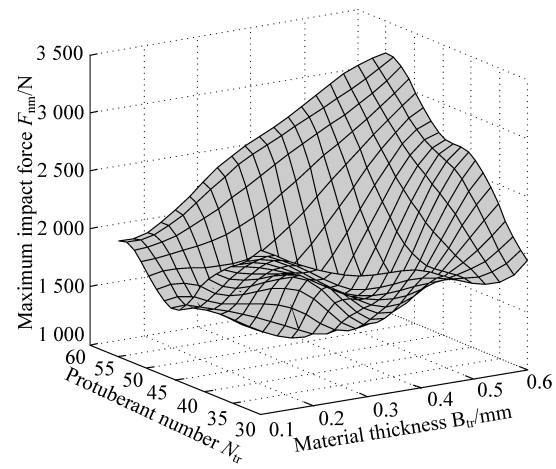

Fig. (6). Influences of tolerance ring parameters on maximum impact forces. 


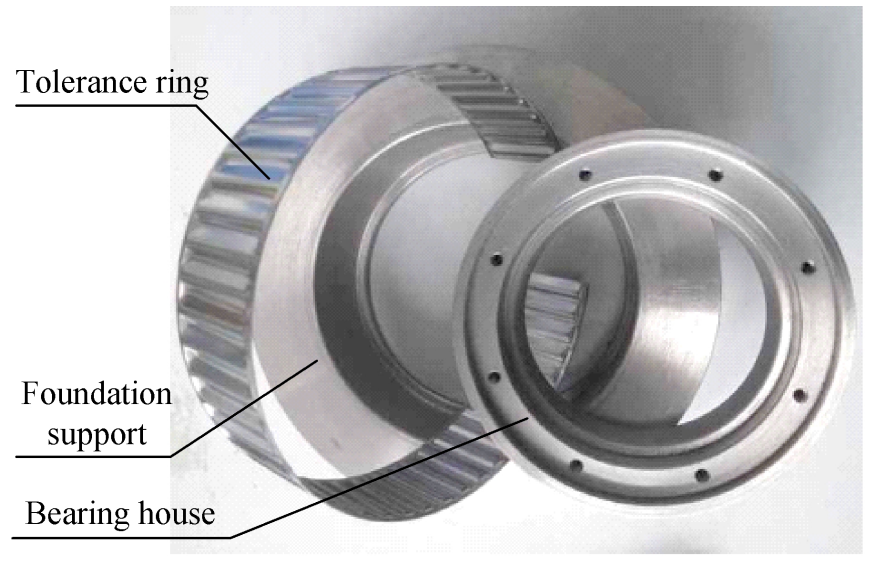

Fig. (7). Parts of analyzed DDCB.

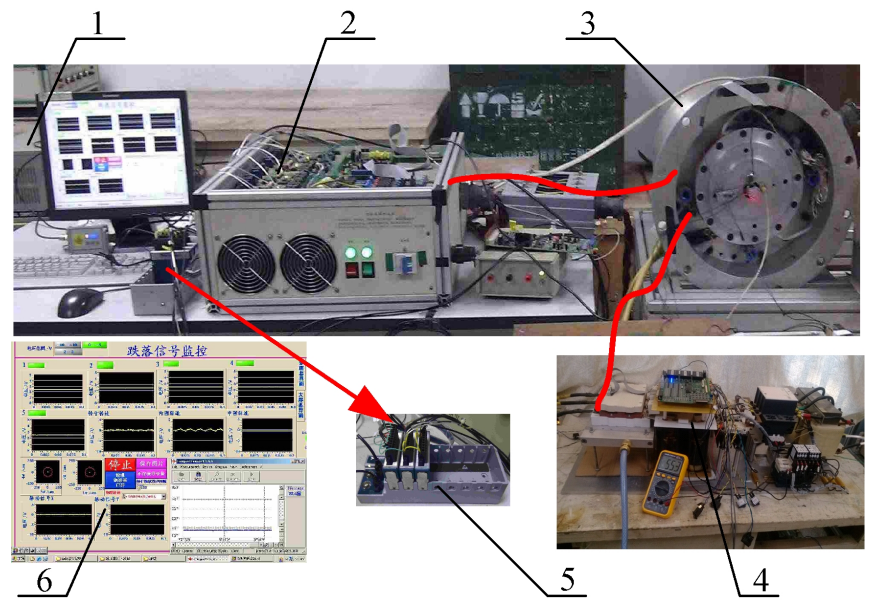

Fig. (8). Rotor drop experiment rig.

\section{CONCLUSION}

In this paper, the new type DDCB with the support of tolerance ring is proposed. The optimum tolerance ring design parameters are obtained through simulations. The following conclusions can be obtained:

1. It is necessary to establish dynamic models to obtain proper design parameters of tolerance ring before manufacture.

2. Using proper tolerance ring helps to reduce the rotor vibration amplitudes and impact forces after rotor drop.

\section{CONFLICT OF INTEREST}

The authors confirm that this article content has no conflict of interest.

\section{ACKNOWLEDGEMENTS}

This work was financially supported by National Natural Science Foundation of China (51405040); the Applied Basic Research in Chanzhou city of China (CJ20140048). (a) Without tolerance ring

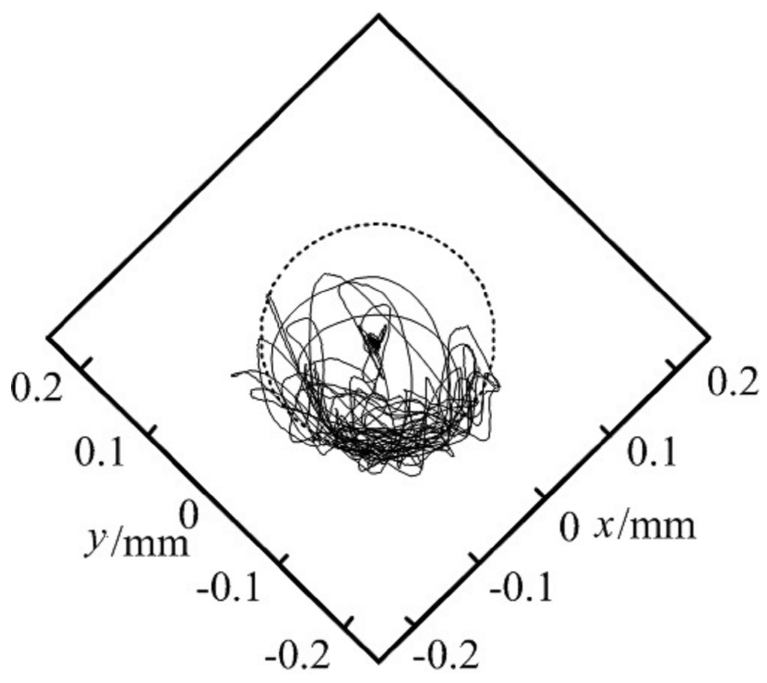

(b) Type "A"

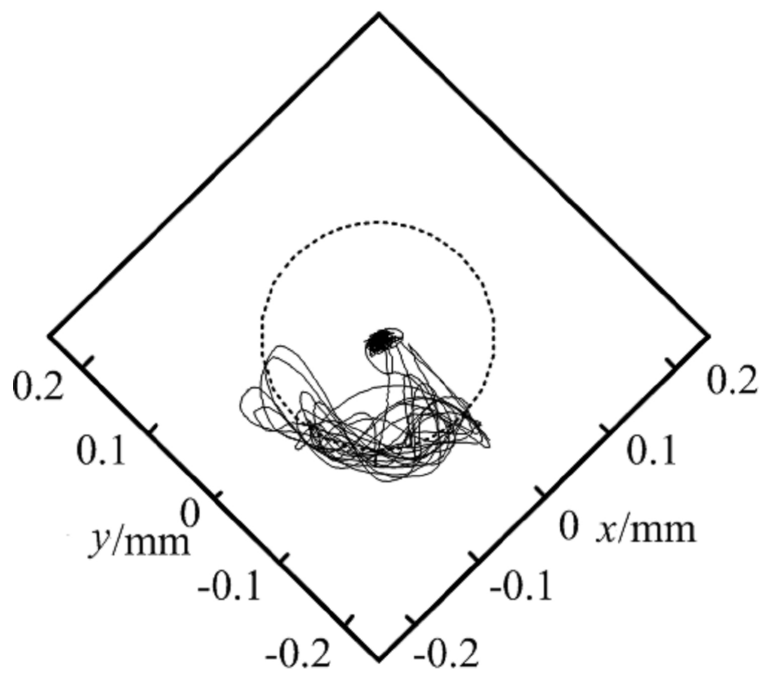

(c) Type "B"

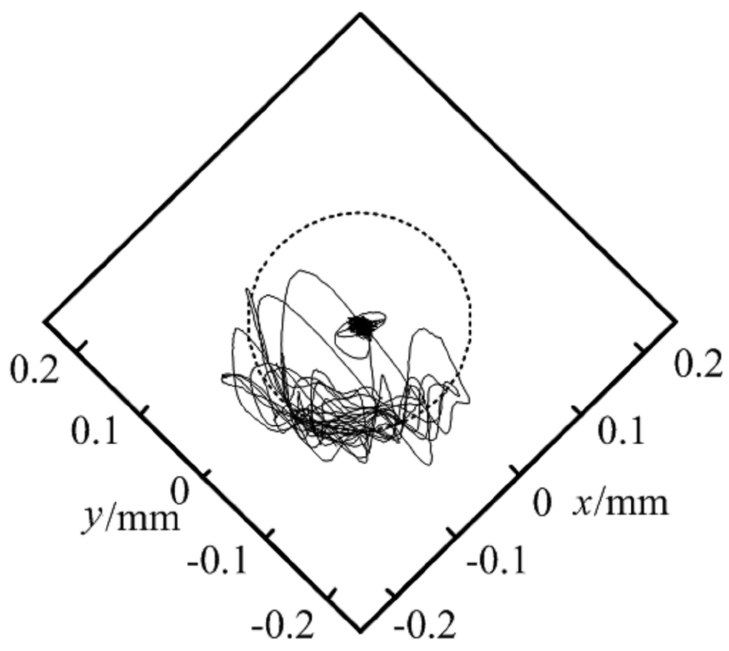

Fig. (9). Rotor orbits obtained by experiments. 


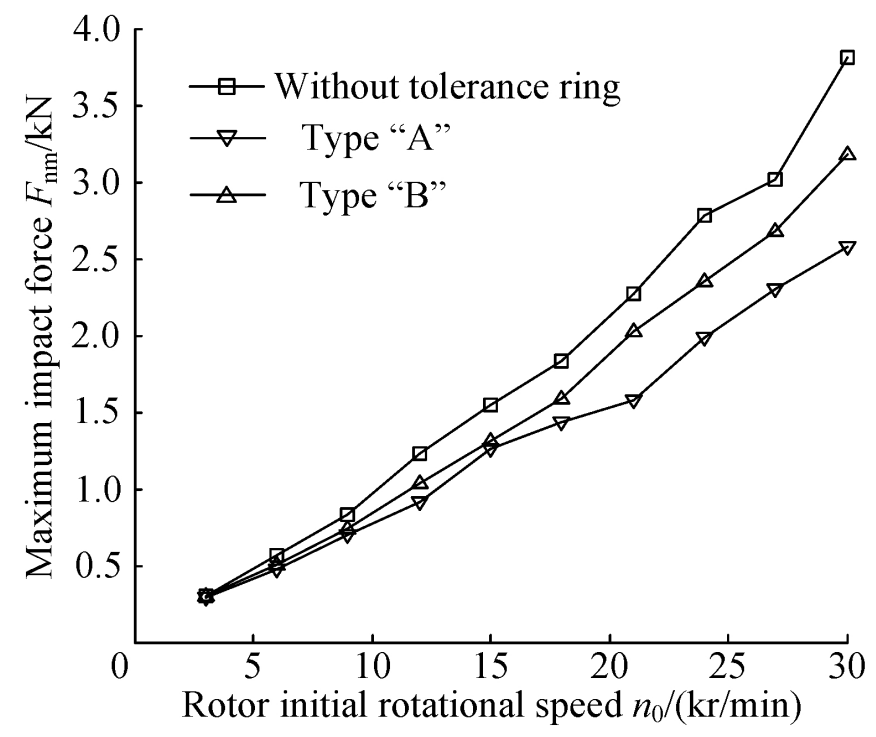

Fig. (10). Maximum impact force obtained by experiments.

\section{REFERENCES}

[1] H. Xie, and G.T. Flowers, "Steady-state dynamic behavior of an auxiliary bearing supported rotor system," In: Proceedings of American Society of Mechanical Engineers Winter Annual Meeting, Chicago, USA, 1994, pp. 1-11.

[2] M.O.T. Cole, P.S. Keogh, and C.R. Burrows, "The dynamic behavior of a rolling element auxiliary bearing following rotor impact, " Journal of Tribology, vol. 124, pp. 406-606, 2002.

[3] X. Wang, and S. Noah, "Nonlinear dynamics of a magnetically supported rotor on safety auxiliary bearings," Journal of Vibration and Acoustics, vol. 120, pp. 596-606, 1998.

[4] M. Salehi, and H. Heshmat, "On the dynamic and thermal performance of a zero clearance auxiliary bearing (zcab) for a magnetic bearing system," Journal of Tribology, vol. 43, pp. 435440, 2008.

[5] D. Kim, and M. K Varrey, "Imbalance response and stability characteristics of a rotor supported by hybrid air foil bearings," Journal of Tribology, vol. 55, no. 4, pp. 529-538, 2012.

[6] Y. L. Zhu, C. W. Jin, and L. X Xu, "Dynamic response of rotor drops onto double-decker catcher bearing," Chinese Journal of Mechanical Engineering, vol. 26, no. 1, pp. 104-113, 2013.

[7] J. W. Smith, "Tolerance Rings (Presenting examples of MotorOverload-Protection applications from the Appliance and Automotive industries), "IEEE Industry Applications Magazine, vol. 8, no. 5, pp. 74-82, 2002.

[8] H. Hertz, "On the contact of elastic solids, "Journal Für die Reine und Angewandte Mathematik, vol. 1881, pp.20-35, 1881. 